\title{
Haiti's earthquake: a multiprofessional experience
}

\author{
Terremoto no Haiti: uma experiência multiprofissional
}

\author{
Milton Steinman ${ }^{1}$, Melissa Simon Gumera ${ }^{1}$, Mario Ferretti ${ }^{1}$, Cristiane Isabela de Almeida ${ }^{1}$, \\ Maria Tereza Augusto Ioshimoto ${ }^{1}$, Silvia Gusman ${ }^{1}$, Miguel Cendoroglo Neto ${ }^{1}$, Oscar Fernando Pavão dos Santos ${ }^{1}$, \\ Alberto Hideki Kanamura ${ }^{2}$, Claudio Luiz Lottenberg ${ }^{3}$
}

\section{INTRODUCTION}

On January 12, 2010 an earthquake of 7.0 on the Richter scale struck Haiti, the poorest country in America. The quake's epicenter was in Leogane with extension to almost all the country also hitting Portau-Prince, the country's capital. This disaster caused a huge destruction and devastated more than 250,000 of houses and commercial buildings and left more than a million of homeless people. In the aftermath, this catastrophe caused 230,000 deaths and more than 30,000 wounded people ${ }^{(1)}$. (Figures $1 \mathrm{~A}$ and B) After the disaster medical institutions, government and non-governmental organizations from around the world geared up to help. Many volunteer health professionals from different areas came together to perform this task.

Previous studies described the severity and epidemiology of injuries post-earthquakes ${ }^{(2-3)}$. The Wenchuan 2008 earthquake in China presented, based on the Injury Severity Score (ISS), $45 \%$ of cases with less than eight injuries (minor injuries), $41 \%$ between 9 and 14 (moderate injuries) and $13.9 \%$ over 15 (severe injuries) ${ }^{(2)}$. Of the injuries of the Kashemir 2005 earthquake in Pakistan, 64.9\% were superficial lacerations, $22.2 \%$ were fractures and $5.9 \%$ were contusions and sprains ${ }^{(3)}$. These studies pointed out the need of a coordinated action and a well equipped hospital to take care of the victims.

We believe that huge disasters are the greatest threat facing mankind. On account of Haiti limited resources and the great number of patients who needed care, a multi-professional action after the earthquake became
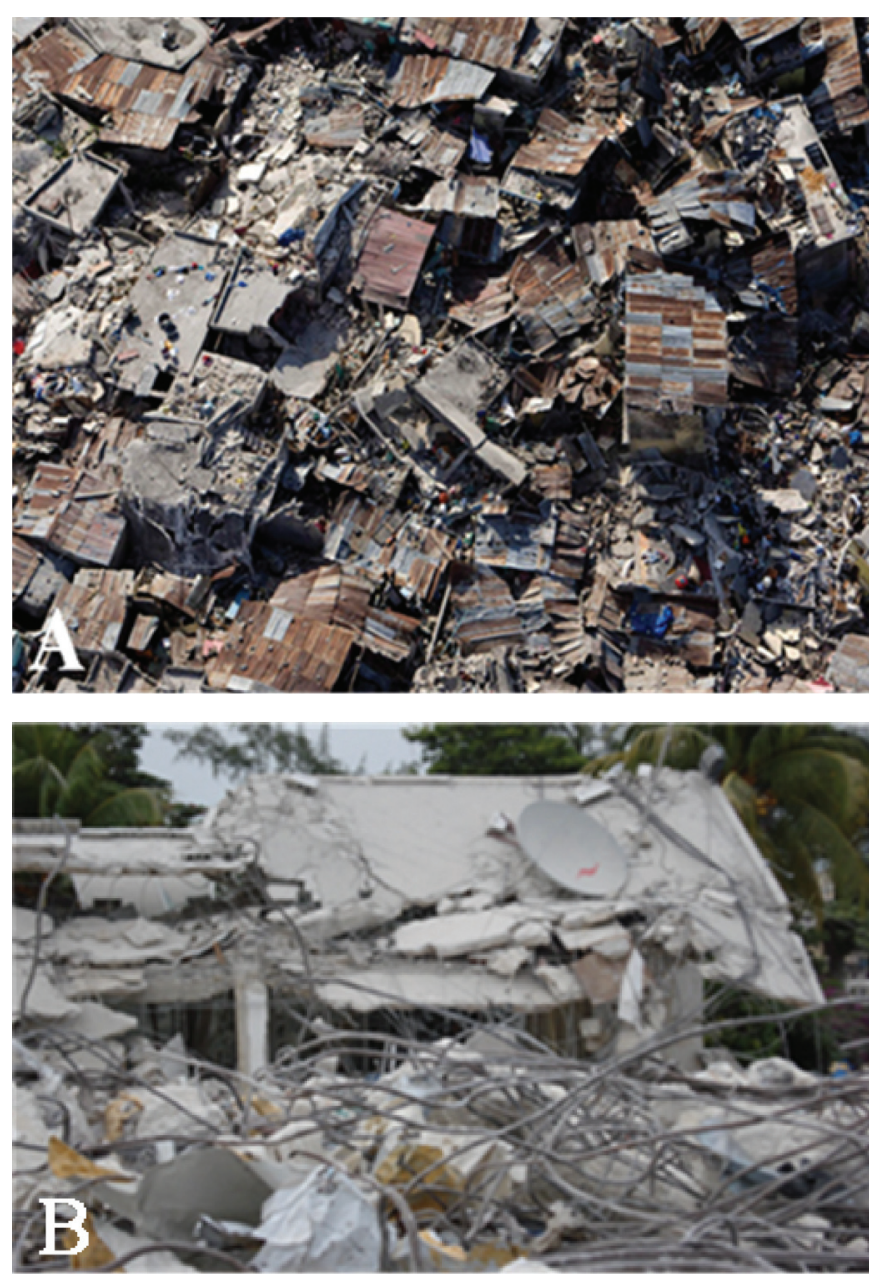

Figura 1. A. Aerial view of a popular housing development after the earthquake. B. Example of destruction of a building after the earthquake

\footnotetext{
This study was carried out at Hospital Israelita Albert Einstein - HIAE - São Paulo (SP), Brasil.

${ }^{1}$ Hospital Israelita Albert Einstein - HIAE, São Paulo (SP), Brazil.

${ }^{2}$ Instituto Israelita de Responsabilidade Social da Sociedade Beneficente Israelita Brasileira Albert Einstein - SBIBAE, São Paulo (SP), Brazil.

${ }^{3}$ Sociedade Beneficente Israelita Brasileira Albert Einstein - SBIBAE, São Paulo (SP), Brazil.

Corresponding author: Milton Steinman - Avenida Albert Einstein, 627 - Consultório 116B, Bloco A1 - $1^{0}$ andar - Morumbi - CEP 05651 -901 - São Paulo (SP), Brasil - Tel.: 112151 -0116 e-mail: miltons@einstein.br

Received: Aug 1, 2010 - Accepted: Dec 8, 2010

* There is no conflict of interest
} 
crucial to deliver adequate medical care. Some studies reported the multi-professional action of specific groups after this quake, superficially showing organizational aspects, dividing experiences and learned lessons $(4,5)$. However, few data presented results of multiprofessional team actions after earthquakes.

Because of the diversity of clinical pictures after catastrophes we believe that a multi-professional team can promote better medical care and improve humanitarian service in such situations.

We aim to describe qualitative and quantitatively the humanitarian and multi-professional action of Hospital Israelita Albert Einstein (HIAE) to assist the victims of the Haiti earthquake that enrolled surgeons, orthopedist, psychologists, physiatrists, occupational therapists, physiotherapists and managers.

\section{ACTIONS}

\section{Organization, planning and early action}

Hours after the earthquake, HIAE a Brazilian beneficent organization, offered to the devastated country a prompt and consistent response. To plan the actions we promptly organized a meeting including several areas of the hospital. Despite the fact that, at that moment, we did not have data on the medical consequences of the disaster, our board of directors, and the departments of critical care, radiology, nursing, pharmacy, engineering, the laboratory, the surgical center and human resources made a list of the resources that could be useful in such a catastrophic situation.

Few hours later a multi-professional team was set up and a huge amount of equipment and critical care medications were made available. Adequate measures were taken so that all participants were vaccinated against yellow fever, hepatitis and tetanus.

Because of the logistical difficulties and because the airport of Port-au-Prince, hit by the quake, had been closed, we decided to send at first a medical team to get the exact diagnosis of the situation, to choose a workspace in order to fulfill our sustainability plan and to deliver medical care.

A group of leaders of our medical and social responsibility areas worked together to give logistic support for the physicians that were sent abroad and also to set up diplomatic issues in order to perform the main action.

\section{The main action}

Our main action was to send a multi-professional team to the previously appointed place to attend the earthquake victims.
The teams were set up according to demands and needs. Initially, physicians, nursing, laboratory technicians, pharmaceutics and maintenance engineers were sent. The subsequent teams were set up based on these previous ones.

Our proposal was to stay for 40 days in alternate days of labor, rotating every 10 to 15 days of activities.

We decided to send younger volunteer professionals with an adequate psychological profile, skilled and ready to face any adverse emergency situation.

\section{The multi-professional action}

The multi-professional team was composed by general surgeons, orthopedists, anesthesiologists, pediatricians, obstetricians, primary physicians and intensivists. The rehabilitation team included physiatricians, occupational therapists, psychologists and physiotherapists. The nursing team worked together with the medical team at the surgical center and with the rehabilitation team. A support group was composed by maintenance technicians, pharmaceutics. Laboratory and radiology technicians were also included.

Our multi-professional team had the goal to deliver direct care to a specific group of patients, as well as to attend the requests from the camp. Activities began daily at 7 a.m. after a meeting with the camp coordination to update the professionals on the activities, admission and discharge of patients.

- Clinical action: patients were assessed daily by using clinical data and prescriptions were made and put into practice together with the nursing team.

- Surgical action: included visits to patients to apply or remove wound dressings and to schedule the procedures that needed sedation and anesthesia in the camp surgical centers. Eventual interappointments were also performed.

- Orthopedic action: the camp orthopedic team had one HIAE orthopedist, one orthopedist of the University of Chicago and two more orthopedists of a charity organization named Operation Smile. The Operation Smile had two mobile operating rooms and a team of anesthesiologists and nurses. The orthopedic action was intended to make a complete diagnosis of skeletal and muscle lesions in the camp patients and then decide upon the adequate procedures.

The diagnosis of lesions was made considering the previous history of the current disease and the patient's physical examination both performed inside the tents. If a radiography was needed, it was done using a hand- 
held portable X-ray machine (Nomad Pro, Aribex , USA) which is habitually used to X-ray dental or small bones of animals.

After a diagnosis was made at the evaluation moment, treatments which did not require a surgical intervention were classified as satisfactory and those that needed immediate or late surgical treatment were classified as unsatisfactory.

- Pediatric action: was performed by clinical evaluation and inter-appointments performed in the tents and in the screening area.

- Nursing team action: this action was performed by broad interaction with the other teams. The nursing team was daily attending all the camp patients according to the needs from the tents, delivering direct care, giving support at the operating rooms, to the pharmacy or to the rehabilitation teams.

- Rehabilitation team action: the evaluation of patients included diagnosis and prognosis of the handicaped, prescription of prosthesis, and locomotor rehabilitation equipments, therapeutic plans for physical rehabilitation and a local workspace for organization duties (either internal or external) together with other therapists (physiotherapists, occupational therapists and psychologists). These professionals also prescribed medication for acupuncture and auricular-acupuncture, and gave support to the nursing team.

- Physicological action: evaluation and psychological interventions were performed focusing on symptoms of post-traumatic stress disorders of patients and their families, some playful activities were done everyday with children (either patients or patients' children). These professionals also supported the nursing team.

\section{RESULTS}

According to the described actions, we highlight the following results.

\section{Organization, planning and previous action}

About a hundred professionals who were willing to participate at any time in the mission were gathered through volunteer mobilization.

The initial period lasted 10 days and was characterized by visits and work at different places between the Jimani county bordering the Dominican Republic and Port-au-Prince, besides a number of contacts with governmental organizations. The Love a Child orphanage at Fond Parisien, $37 \mathrm{~km}$ from Portau-Prince was chosen as our headquarters, because this place offered safety and basic conditions for the team.
During this initial period it was possible to identify the most frequent diagnosis and figure out elements to set up teams and elaborate the needs for material resources.

Because the official language of the country is French and the majority of Haitians speak creole only, we had a translator during the day time.

A total of 52 professionals had the opportunity to take part in this mission.

\section{The main action}

Two hundred and forty patients were distributed among the various care teams (from The United States of America, Ecuador and Brazil). Our group was responsible for 40 patients placed in tents designed for two or three patients each (Figures 2A, B and C). However, our action covered all patients according to the diversity of the professionals in the groups and the huge demand of victims requiring care.

The official records of care included: the clinical picture, the daily planning, an electronic prescription and drug delivery. Every four days a medical and nursing team was on duty overnight to give support in case of the occurrence of an emergency at the camp.

The patients' mean age was 27.00 years +19.88 (Figure 3), most of them were women (80\%). Regarding schooling, 55\% had the equivalent to Brazilian's basic education. Among patients the most common physical injuries were fractures of lower limbs followed by contusions and sprains. (Figure 4)

Of the 40 patients under our responsibility, 13 had suffered amputations mainly on the left lower limb (Figure 5).

\section{Activities}

The team activities included visits to all patients, prescription and drug administration, wound dressing changes and specific actions depending on the special care needed.

- Clinical activity: two visits were done daily. All patients were visited in the morning to evaluate their follow-up and prescription and in the afternoon, to verify pending problems and to re-evaluate more severe cases.

- Surgical activity: twenty per cent of patients required wound dressings which were performed daily under medical supervision inside the tents. One to two surgical procedures were carried out in the surgical center, using narcosis or sympathetic block. The most common diagnosis were: infected wounds, decubitus ulcers and reconstruction of amputation stumps. 

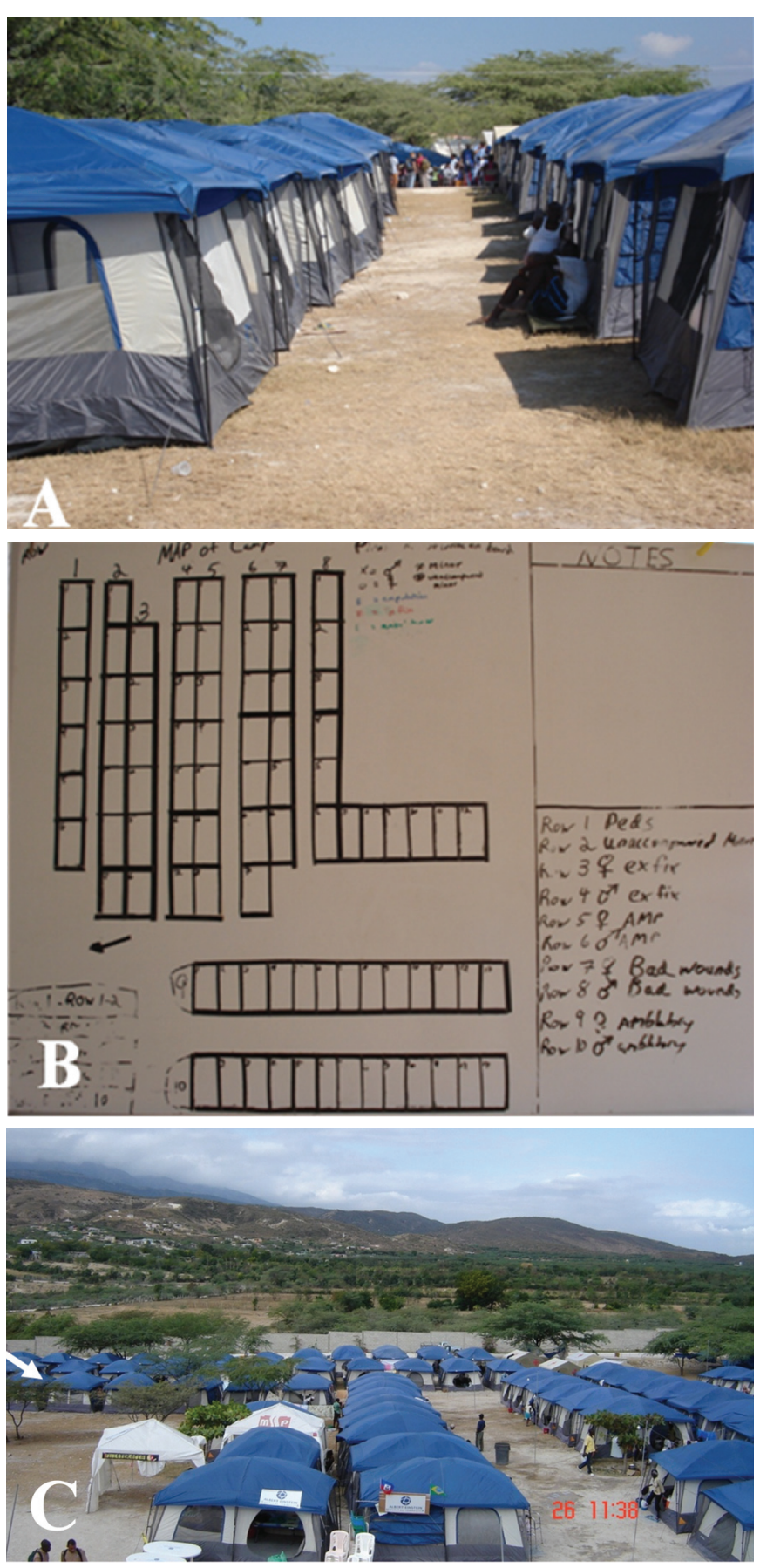

Figura 2. A. View of the tents. B. Camp map with the tents positioned as they were ordered. C. Camp map with the tents (in reverse $L$ ) under the responsibility of HIAE (arrow)

- Orthopedic activity: the number of patients assessed by our orthopedist exceeded the initial number assigned to us (40 patients). In fact, the orthopedist assessed 103 patients, being $59(57.3 \%)$ women and $44(42.7 \%)$ men. These patients' mean age was 28 years, varying from 2 to 82 years. From this total, 40 patients $(38.8 \%)$ were under 18 years of age and only $10(9.7 \%)$ were more than 60 years old. The majority

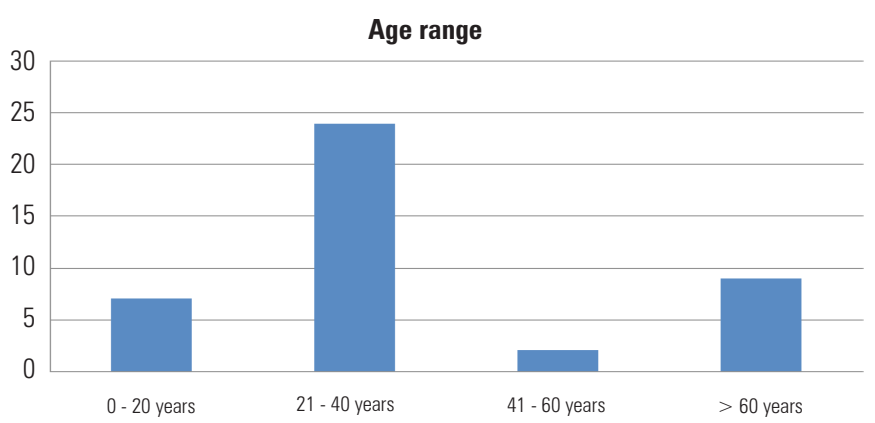

Figure 3. Age distribution

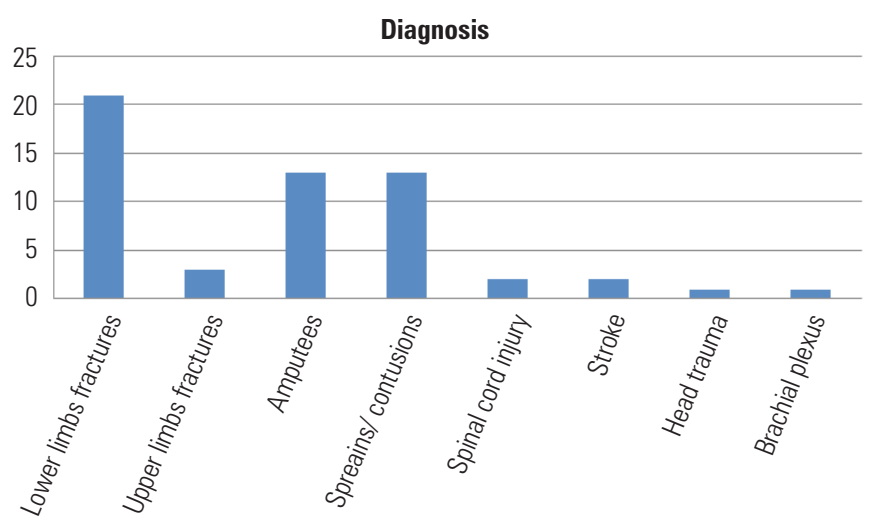

Figure 4. Injury distribution in 40 pacients



Figure 5. Distribution of the amputation level

of patients had already been treated, however, after three weeks of injuries' occurrence, a census describing the complete orthopedic diagnosis and the adequate behavior was not yet been performed.

Of the 103 cases, $79(76.6 \%)$ were fractures, $3(2.9 \%)$ dislocation or severe sprains and $18(18 \%)$ were cases of amputation. After the orthopedic evaluation, 71 patients $(68.9 \%)$ were considered satisfactorily treated and 32 patients $(31.06 \%)$ unsatisfactorily. Among these 
32 cases, 10 required early surgery and the other 22 cases had vicious consolidation of fractures (shortening or angular deviations), or late consolidation of fractures because the inadequate treatment previously given (Figure 6 A, B, C e D). Six children who had diaphyseal femur fracture and one child with hip dislocation had the spica cast removed after five weeks of the injury. One child with fracture of tibia also had the plaster removed.

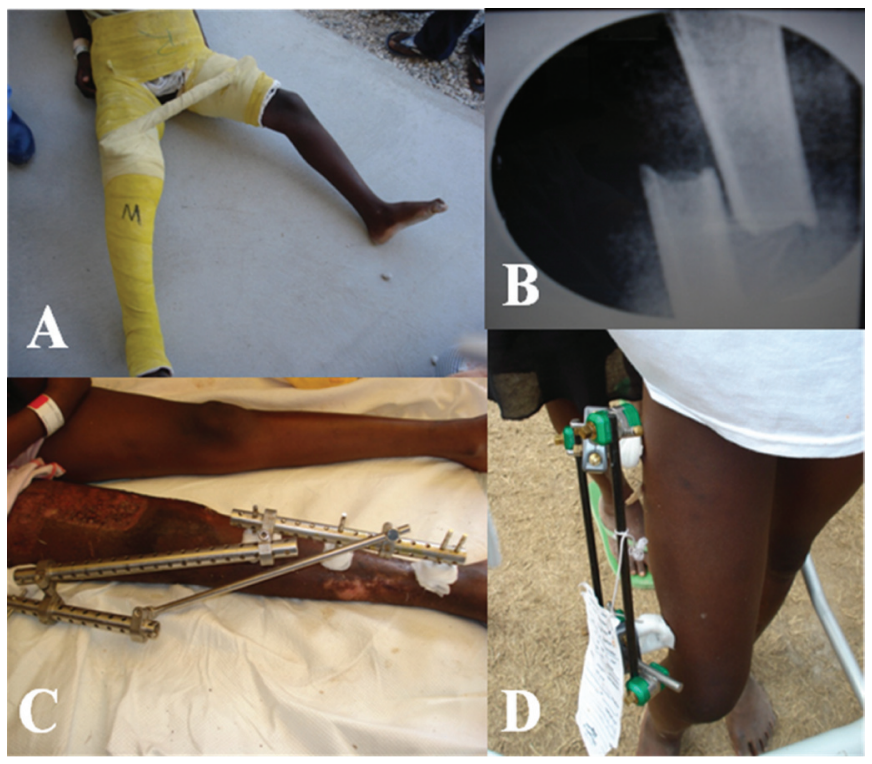

A. Child with diaphyseal femoral fracture with a spica cast. B. Shortening of a diaphyseal femoral fracture in a 18 year old girl, an unacceptable case. C. A case of disphyseal tibial and femoral (floating knee) with skin lesions. D.

Patient with a diaphyseal femoral fracture inadequately treated because the fixator did not provide enough stability for weight discharge.

However, the fracture was aligned and was considered satisfactory for the moment.

Figura 6. Orthopedic patients

- Nursing activities: the nursing team performed evaluations everyday, drug administration and insertion of peripheral venous access. All 40 patients received advice on skin hydration and hands cleaning. Bladder and bowel training was needed for spinal cord injury patients.

- Rehabilitation team activities: all victims of the quake required some kind of rehabilitation. More than three weeks after the disaster many patients were staying in their tents for several days, and immobilism become a common characteristic either related or not to trauma. The patients under our responsibility were evaluated in relation to this aspect and we also assisted patients from the other teams.

This work included: diagnosis and prognosis of disabilities, prescription of prosthesis, locomotor rehabilitation equipments and a therapeutic plan of physical rehabilitation. We organized sites for care (both internal and external). Drugs for acupuncture and auricular-acupuncture were prescribed, and in this latter case, the care team was also involved. An outside area for group therapy, living and leisure was created (Figure 7).

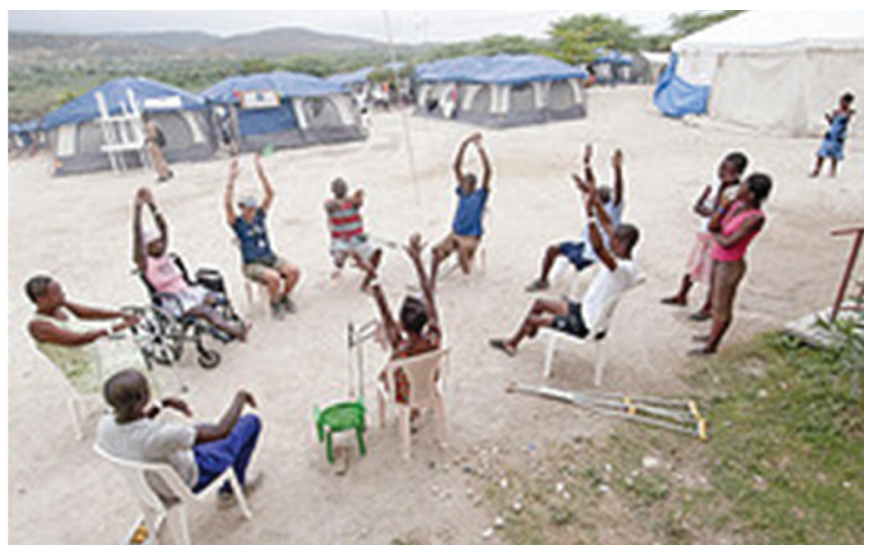

Figure 7. Group of amputees realizing group physiotherapy

The physical rehabilitation team (physiotherapy and occupational therapy) treated the 40 patients with single or multiple sequels of fractures of lower limbs and upper limbs, brachial plexus injury, traumatic injury without fractured limbs, head trauma and patients who underwent amputations.

All professionals frequently stimulated the patients to leave their tents to joint social activities.

- Psychological evaluation: patients who presented symptoms of post-traumatic stress disorders were submitted to psychological interventions daily besides playful activities with children (either patients or patients' children). Some dynamics were performed with teenagers who survived from the crumbling of an orphanage in Port-au-Prince. From the total, $5 \%$ reported traumatic memories on the disaster moment. After clinical and psychological evaluation we identified that 22 patients $(55 \%)$ have symptoms of depression and $6(40 \%)$ of anxiety.

\section{COMMENTS}

In a massive earthquake of such territorial proportion it is impossible to calculate losses. The disrupting of communication systems, the difficulties to access the quake local, the collapse of health buildings and the scarcity of water, food and power associated to the great number of wounded turned the humanitarian mission extremely difficult to handle ${ }^{(2)}$. 
Although we did not have previous experience in earthquakes, other previous accomplishments of HIAE facing epidemics (meningococcal meningitis in the 70's and dengue in 2008) our philanthropic basis motivated us to respond to the disaster. A private organization of excellence that has a large skilled staff could provide a sustainable voluntary response in disasters. The cooperation with the local government and/or nongovernmental organizations was extremely important for the collaborative actions.

The diagnosis of the situation associated to a continuous interchange of information with our headquarters in Brazil were essential to recruit, select and provide the specialty teams and the resources needed.

To choose a workspace was a critical part of the mission and the following premises were taken into account in this regard: the assurance of the team's safety, the need of a place for basic hygiene and rest, as well as a basic infrastructure with electricity, water and place to perform surgeries. For the aftershocks, a place without edifications was fundamental. In addition, the self-sufficiency in food and water is highly important in such situations of catastrophe ${ }^{(6)}$.

The chosen workspace was adequate because it was near the Dominican Republic border which made easier the arrival of teams and material resources, as the displacement to nearby sites in order to get supplies and food for the team. Besides being a broad place, there was a helipad that was eventually used for patient transfer.

The initial period of evaluation provided information on most common diagnosis: trauma, open and closed fractures, lesions associated to body segments of medium and small complexity, post-operative patients transferred from hospitals in Jimani and Port-au-Prince, and other clinical situations not directly related to the earthquake. This period was extremely important to help to set up subsequent teams.

The length of stay of the mission proposed by HIAE (40 days) involved different aspects of those habitually seen in the initial phases after massive disasters.

The majority of patients in the camp were homeless $(90 \%)$ and the tents became their home. The collapse of families, the endless number of homelesses and orphans characterized a common profile of the patients.

Besides millions of deaths, the wounded people had single or multi-systemic lesions of diverse complexities that required a broad approach. In addition, emotional sequels started precociously. A multi-professional team can provide an efficient, prompt and coordinated care $^{(3)}$.

To evaluate the quality of care in the first days of a disaster is not an easy job, considering a scenario of huge catastrophe that lacks the necessary resources for orthopedic care $\mathrm{l}^{(7)}$. Patients in the camp were real survivors.

However, many procedures took place at the initial phases and required complementary treatment or revision. The absence or data scarcity on clinical diagnosis and treatment was quite common (Figure 8). When our team started this evaluation we did not have an X-ray machine, and many patients were submitted to a radiologic evaluation for the first time only three weeks after the earthquake.

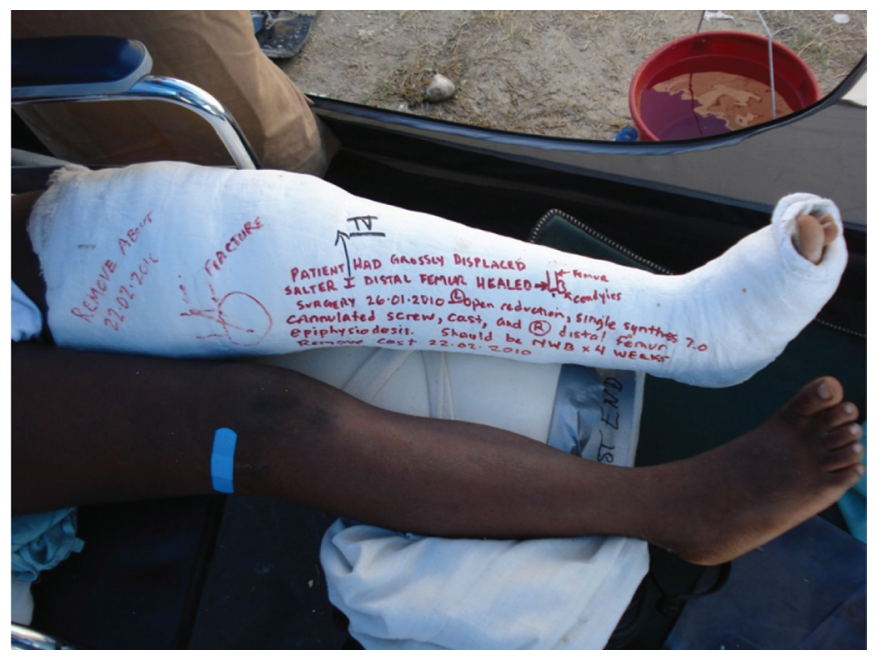

Figure 8. Patient with a cast in the left leg over which descriptive data on the clinical course and management were written

It was not only a post-operative evaluation, as it required a re-screening of cases to separate patients according to additional procedures as needed, so we decided to classify care plainly as satisfactory and unsatisfactory. Probably, the number of orthopedic lesions not appropriately treated was higher than the satisfactory ones. However, in such an adverse situation and for the scarcity of available resources for surgical procedures, this management was the best way we have found to keep treatments going on. A subsequent analysis will be fundamental to evaluate the functional results.

Among the challenges this catastrophe brought, delivering health care to the survivors was the most problematic situation ${ }^{(4,5)}$. The complexity and variety of cases required different answers that had to consider not only the clinical aspects, but also the social, psychological and economical impacts. Indeed, a preview of demands allows the development of a working process which could be better accomplished in a cooperative way among the various professionals who were involved.

A prompt response has a huge impact to the victim's recovery after an earthquake. In addition, an efficient 
emergency response requires the patient to be in the right place at the right time and in a catastrophic situation this is hard to achieve.

Finally, we verified that demands and needs of patients after an earthquake may vary broadly day by day, which implies that the teams and the place for care delivery must have as maximum as possible flexibility and versatility. The medical priorities can change from actions to preserve life to procedures to save limbs of amputations or start rehabilitation, from a debridement to nursing care of wounds, from clinical treatment to psychological support. Therefore, we believe that the multi-professional approach constitutes the ultimate strategy to delivery care for victims after an earthquake.

\section{ACKNOWLEDGES}

This paper would not be possible to accomplish without idealization, planning, collaboration and actions done by thishuge teamofProfessionalfromSociedadeBeneficente Israelita Brasileira Albert Einstein (SBIBAE) whom we would like to acknowledge: Nelson Akamine; Luciana Guastelli; Wladimir Mendes Borges Filho; Alex Sandro Gomes; Dov Smaletz; Valdeci Alves Ferreira; Renato P. da Conceição; Patricia Sousa Machado; Ana Carolina Martins Pereira da Silva; Barbara de Oliveira Manoel; Carolina SantAnna Azevedo; Claudia Candido da Luz; Clesio Nepomuceno; Joper Fonseca Jr; Marcelo de Oliveira Anhê; Maria Christina M. A. Fleury; Maria Roza de Jesus S. Oliveira; Mariana Perroni de Oliveira; Reginaldo Rogerio de Campos; Ronildo Galdino Guimaraes; Rosana Ravagnani Campedelli;
Sandra Cristina Shiramizo; Tais Rodrigues de Lara; Tania Maria Russo Zamataro; William Abrão Saad Jr; Felipe Camilo Val; Coroacy dos Santos Jr; Débora Puntel; Fabio Jorge Racy; Graziele de Paula Jimenes; Gustavo Janot; Haggeas Fernandes; Jorge Luis Saraiva; Luiz Alexandre de Castro; Marcele Pesavento; Maria Paula Vilela; Michael Medeiros; Tatiana Castagnari; Adriana Ferreira; Adriana Marcos; Alexandre Marra; Arnaldo Felix; Daniela Takito; Eduardo Cordioli; Fabio Ferracini; Isaura Maria; Marcilio Mendonça; Marcio Damascena; Maria Beatriz Perondi; Mauro Ribas; Thais Caprera Carvalho; Waldenira Rocha.

\section{REFERENCES}

1. Gamulin A, Villiger Y, Hagon 0. [Disaster medicine: mission in Haiti]. Rev Med Suisse. 2010;6(248):973-7.

2. Yang $\mathrm{C}$, Wang HY, Zhong HJ, Zhou L, Jiang DM, Du DY, et al. The epidemiological analyses of trauma patients in Chongqing teaching hospitals following the Wenchuan earthquake. Injury. 2009;40(5):488-92.

3. Mulvey JM, Awan SU, Qadri AA, Maqsood MA. Profile of injuries arising from the 2005 Kashmir earthquake: the first 72 h. Injury. 2008;39(5): $554-60$.

4. Jaffer AK, Campo RE, Gaski G, Reyes M, Gebhard R, Ginzburg E, et al. An Academic centers delivery of care after the Haitian earthquake. Ann Intern Med. 2010;153(4):262-5.

5. Babcock C, Baer C, Bayram JD, Chamberlain S, Chan JL, Galvin S, et al. Chicago medical response to the 2010 earthquake in Haiti: translating academic collaboration into direct humanitarian response. Disaster Med Public Health Prep. 2010;4(2):169-73.

6. Water and sanitation on health emergencies: the role of $\mathrm{WHO}$ to the earthquake in Haiti, 12 January 2010. Wkly Epidemiol Rec. 2010;85(36): 349-54.

7. Hirshberg A, Holcomb J, Mattox K. Hospital trauma care in multiple casualty incidents: A critical view. Ann Emerg Med. 2001;37(6):647-52. 